\title{
The conjoint associations of adherence to physical activity and dietary guidelines with cardiometabolic health: The Framingham Heart Study
}

Joowon Lee

Boston University

Maura E. Walker

Boston University

Maximillian T. Bourdillon

Boston University

Nicole Spartano

Boston University

Gail T. Rogers

Boston University

Paul F. Jacques

Tufts University

Ramachandran S. Vasan

Boston University

Vanessa Xanthakis ( $\sim$ Vanessax@bu.edu )

Boston University School of Medicine

\section{Research}

Keywords: physical activity, diet quality, guidelines, cardiometabolic health

Posted Date: August 2nd, 2020

DOI: https://doi.org/10.21203/rs.3.rs-50754/v1

License: (1) This work is licensed under a Creative Commons Attribution 4.0 International License.

Read Full License

Version of Record: A version of this preprint was published at Journal of the American Heart Association on April 6th, 2021. See the published version at https://doi.org/10.1161/JAHA.120.019800. 


\section{Abstract \\ Background}

The conjoint associations of adherence to the recent physical activity (PA) and dietary guidelines with the metabolic syndrome (MetS) are incompletely understood.

\section{Methods}

We evaluated 2,379 Framingham Heart Study Third Generation participants (mean age 47 years, 54.4\% women) attending examination cycle 2 . We examined the cross-sectional relations of adherence to the 2018 Physical Activity Guidelines for Americans (PAG, binary; moderate to vigorous PA [MVPA] $\geq 150$ minutes/week vs. $<150$ minutes/week) and 2015 Dietary Guidelines for Americans (DGA, binary; 2015 DGA adherence Index [DGAI-2015] $\geq$ median vs. <median [score 62.1/100]) with prevalence of the MetS using generalized linear models. We also related adherence to guidelines with the incidence of MetS prospectively, using Cox proportional hazards regression with discrete time intervals.

\section{Results}

Adherence to the 2018 PAG (odds ratio [OR] 0.49, 95\% Cl 0.40-0.60) and 2015 DGA (OR 0.67, 95\% Cl $0.51-0.90$ ) were individually associated with lower odds of prevalent MetS, while conjoint adherence to both guidelines was associated with the lowest odds of MetS (OR $0.35,95 \% \mathrm{Cl} 0.26-0.47$ ) compared to the referent group (non-adherence to both guidelines). Adherence to the 2018 PAG (hazards ratio [HR] $0.66,95 \% \mathrm{Cl} 0.50-0.88)$ and $2015 \mathrm{DGA}(\mathrm{HR} 0.68,95 \% \mathrm{Cl} 0.51-0.90)$ were associated with lower risk of MetS, prospectively. Additionally, we observed a $52 \%$ lower risk of MetS in individuals who adhered to both guidelines compared to the referent group.

\section{Conclusions}

Maintaining both regular physical activity and a healthy diet in midlife may be required for optimal cardiometabolic health in later life.

\section{Background}

The metabolic syndrome (MetS) is the clustering of key cardiometabolic risk factors, such as abdominal obesity, insulin resistance, hyperglycemia, dyslipidemia, and high blood pressure [1]. The presence of MetS is a major risk factor for type 2 diabetes and cardiovascular disease (CVD) [2, 3]. However, evidence indicates that healthy lifestyle behaviors are associated with favorable cardiometabolic health [4]. Additionally, favorable cardiometabolic health in middle-adulthood has been associated with lower 
disease burden later in life [5]. Thus, adherence to healthy lifestyle modifications in middle-adulthood is a feasible approach to improve cardiometabolic health later in life.

The 2018 Physical Activity Guidelines for Americans (PAG) recommend that adults (18 years and older) achieve a minimum of 150 minutes of moderate-to-vigorous physical activity (MVPA) per week to lower the burden of chronic disease [6]. Likewise, the 2015 Dietary Guidelines for Americans (DGA) suggest adhering to a high-quality healthy dietary pattern for the prevention of chronic disease [7]. Prior studies have reported that time spent in objectively-assessed MVPA [8, 9] and adherence to DGA $[10,11]$, quantified by the 2005 DGA Adherence Index (DGAI), are individually associated with lower odds of MetS in middle-aged adults. The independent associations of PA and diet quality with cardiometabolic health are likely due to shared biological and behavioral mechanisms. However, it is unclear whether adherence to both recent PAG and DGA (as opposed to one or the other) in midlife confers the most favorable cardiometabolic health later in life.

We hypothesized that adherence to both the 2018 PAG and the 2015 DGA will be associated with lower odds of prevalent MetS cross-sectionally, and with a lower risk of developing MetS prospectively.

\section{Methods}

\section{Study design and sample}

We evaluated participants from the Framingham Heart Study (FHS) Third Generation cohort who attended the second examination cycle (2008-2011). The study design and methods of this FHS cohort have been described elsewhere [12]. For the current investigation, we examined two analytical samples: of the 3,411 participants who attended the second examination cycle, 1,032 participants were excluded for the following reasons: refusal to wear an accelerometer or invalid PA data $(n=866)$; unavailable dietary data $(n=150)$; unavailable data on components of MetS $(n=13)$; and missing covariates $(n=3)$, resulting in a sample size of 2,379 (Sample 1); this sample was used to evaluate the cross-sectional associations of adherence to the 2018 PAG (MVPA $\geq 150$ minutes/week vs. MVPA $<150$ minutes/week) and adherence to the 2015 DGA (DGAl-2015 $\geq$ median [score 62.1/100] vs. DGAI-2015 < median) with presence of MetS cross-sectionally. Next, we excluded 817 participants from Sample 1 because they had prevalent MetS at baseline $(n=496)$ or did not have available data on components of MetS at follow-up (third examination cycle [2016-2019, $n=321]$ ), resulting in a sample size of 1,562 (Sample 2), which was used for evaluating the longitudinal associations of adherence to the 2018 PAG and 2015 DGA (DGAl$2015 \geq$ median [score 63.0/100] vs. DGAl-2015<median) with the incidence of MetS. The study was approved by the Boston University Medical Center institutional review board and all participants provided written informed consent.

\section{Objective assessment of physical activity}

At the second examination cycle, participants were asked to wear an omnidirectional accelerometer (Actical model no. 198-0200-00; Philips Respironics, Murrysville, PA, USA) on the hip for eight days, 24 
hours per day (except when bathing or involved in water activity). This accelerometer records signals within $0.5-3 \mathrm{~Hz}$ and accelerations/decelerations within $0.05-2 \mathrm{~g}$. Recorded signals were grouped into 'counts' during 30-second intervals and stored on the device. Data were analyzed using customized software (Kinesoft, version 3.3.63, Saskatchewan, Canada) and a pre-defined protocol for quality control. Measures from the first day of wear were excluded from the analysis. Accelerometer data were considered valid if the device was worn for $\geq 10$ hours per day for at least 4 out of 7 days. Non-wear time was defined as 60 consecutive minutes of zero counts, allowing for 2-minute interruption periods. Nonwear bouts were removed during data processing. Each minute of wear time was classified using previously established cut-points [13-15]. For the current investigation, MVPA was defined as $\geq 743$ counts per 30-second epoch. We defined adherence to the 2018 PAG as MVPA $\geq 150$ minutes/week in accordance with the 2018 PAG Advisory Committee Scientific Report [6]. For participants with $<7$ days, but $\geq 4$ days of valid wear time, we averaged the measured time spent in MVPA over the valid days and extrapolated it to estimate MVPA over 7 days.

\section{Dietary assessment}

Dietary intake was measured using data from the Harvard semi-quantitative food frequency questionnaire (FFQ) administered at the second examination cycle. The Harvard FFQ measures the typical frequency and consumption of 150 food items over the past year [16]. The validity of the Harvard FFQ was previously assessed using seven-day dietary records [16-17]. We only used FFQs that were considered valid ( $<13$ blank items and estimated daily caloric intake $\geq 600 \mathrm{kcal} / \mathrm{d}$ and $<$ 4,000/4,200 kcal/d for women/men) [18].

\section{Dietary Guidelines Adherence Index (DGAl-2015)}

The DGAI-2015 is designed to measure adherence to the 2015 DGA [7]. Details of the DGAl-2015 have previously been described [19]. The DGAI is composed of two sub-scores: food intake and healthy choice. The food intake sub-score measures intake of 14 food groups (fruit; dark green vegetables; orange and red vegetables; starchy vegetables; other vegetables; grains; dairy; meat, proteins, and eggs; seafood; nuts, seeds, and soy; legumes; empty calories, variety in protein choices; and variety of fruits and vegetables). The healthy choice sub-score measures adherence to 10 consumption recommendations regarding intake levels of food groups and nutrients (amounts of total fat, saturated fat, trans fat, sodium, fiber, alcohol; percentage of protein that is lean, dairy that is low-fat, grains that are whole grain, and fruits that are whole fruits). Adherence to dietary recommendations for each sub-score component is scored as a proportion on a continuous scale of $0-1$ (food intake maximum $=14$ and healthy choice maximum $=10$ ). The final DGAI-2015 score consists of summed component scores that are standardized to a range of 0-

100; $\left(\frac{(\text { total food intake score total healthy choice score }}{24}\right) \times 100=$ final DGAI-2015 score. The maximum DGAI- 2015 score is 100, with a higher score indicating higher diet quality. For the present investigation, we dichotomized DGAI-2015 based on the median and used both binary (DGAl-2015 $\geq$ median vs. DGAl-2015<median) and the continuous DGAl-2015 variable (ranging from 0 to 100).

\section{Metabolic syndrome (MetS)}


Participants who met at least three of the following criteria were considered as having the MetS according to the American Heart Association/National Heart, Lung, and Blood Institute (AHA/NHLBI) guidelines [4]: (1) waist circumference $\geq 40 / 35$ inches (men/women); (2) systolic/diastolic blood pressure $\geq 130 / 85 \mathrm{~mm} \mathrm{Hg}$ or use of antihypertensive medication; (3) fasting glucose $\geq 100 \mathrm{mg} / \mathrm{dL}$ or use of anti-diabetic medication; (4) serum triglycerides $\geq 150 \mathrm{mg} / \mathrm{dL}$ or use of lipid-lowering medication; and (5) HDL-C $\leq 40 / 50 \mathrm{mg} / \mathrm{dL}$ (men/women).

\section{Covariates}

All covariates were collected from routine medical history, physical examination, and laboratory assessment at the second examination cycle. In the current investigation, we included the following covariates: age, sex, accelerometer wear time, number of cigarettes smoked per day, total daily calorie intake, and the prevalence of CVD. Accelerometer wear time was determined by subtracting the non-wear time from a 24-hour period. Total daily calorie intake was derived from the aforementioned 150-item FFQ. Prior history of CVD, including fatal or nonfatal myocardial infarction, unstable angina (the prolonged ischemic episode with documented reversible ST-segment changes), peripheral vascular disease (intermittent claudication), cerebrovascular disease (ischemic or hemorrhagic stroke or transient ischemic attack), or heart failure was collected by medical history questionnaire, physical examination, and hospitalization records. All events were adjudicated by a panel of FHS physicians based on previously reported criteria [20].

\section{Statistical analysis}

Age- and sex-adjusted linear regression models were used to evaluate the association between adherence to the 2018 PAG (MVPA $\geq 150$ minutes/week or MVPA $<150$ minutes/week, independent variable) and the continuous DGAl-2015 (dependent variable). Participants were cross-classified by using the binary DGAI-2015 $\geq$ median vs. DGAl-2015 < median and MVPA $\geq 150$ minutes/week vs. MVPA $<150$ minutes/week variables. We used multivariable-adjusted generalized linear models (SAS PROC GENMOD) to evaluate the individual associations of adherence to the 2018 PAG and 2015 DGA (independent variables, separate model for each) with the prevalence of MetS (dependent variable), accounting for familial relatedness. To examine the longitudinal associations of adherence to the 2018 PAG and 2015 DGA with the incident MetS. We modeled MVPA as a binary variable (MVPA $\geq 150$ minutes/week vs. MVPA $<150$ minutes/week [referent]) and as a continuous variable. Adherence to the 2015 DGA was also modeled as a binary variable (DGAl-2015 $\geq$ median vs. DGAl-2015 < median), and a continuous variable. We used Cox proportional hazards regression models with discrete time intervals to evaluate the individual associations of adherence to the 2018 PAG and 2015 DGA (independent variables, separate model for each) with risk of MetS, adjusting for the same covariates at baseline. We confirmed the proportional hazards assumption by including an interaction term between log-time and each exposure variable (MVPA or DGAl-2015) in the Cox regression models.

We also tested a significant multiplicative interaction between MVPA and DGAI-2015 on the association of each with the risk of MetS (both cross-sectionally and longitudinally) by incorporating cross-product 
terms in the multivariable-adjusted models. Additionally, we created four cross-classification groups using adherence to 2018 PAG and 2015 DGA (independent variable, MVPA $\geq 150$ minutes/week vs. $<150$ minutes/week [2 groups] $\times$ DGAl-2015 $\geq$ median vs. DGAl-2015< median [2 groups]) to evaluate the crosssectional and longitudinal associations of conjoint adherence to the 2018 PAG and the 2015 DGA with odds and risk of developing MetS. Participants who did not meet the 2018 PAG $(<150$ minutes/week of MVPA) and had poor adherence to the 2015 DGA (DGAl-2015 < median) were classified as the referent group. All models were adjusted for age, sex, accelerometer wear time, number of cigarettes smoked per day, total daily caloric intake, and prevalence of CVD. As a sensitivity analysis, we categorized DGAI-2015 into three groups based on tertile and examine the association of 2015 DGA (tertile of DGAl-2015) with odds and incidence of MetS to evaluate dose-response relation in which participants who met the 2018 PAG (MVPA $\geq 150$ minutes/week) had progressively lower odds or risk of MetS with increasing adherence to the 2015 DGA.

A two-sided value of $P<0.05$ was considered statistically significant for all models while the value of $P<$ 0.10 was used to determine statistically significant multiplicative interaction. All analyses were performed using SAS software version 9.4 (SAS Institute Inc, Cary, NC).

\section{Results}

\section{Participant characteristics}

The baseline characteristics by sex are presented in Table 1. The average time spent in MVPA was $27.4 \pm$ 20.7 minutes/day and $52.7 \%$ of participants met the criteria of the 2018 PAG (MVPA $\geq 150$ minutes/week). The average scores of the DGAl-2015 were 61.1 (52.1 [DGAl-2015< median, poor adherence], and 70.0 [DGAl-2015 $\geq$ median, higher adherence]. Only $28 \%$ of participants where characterized as both meeting the 2018 PAG and having higher adherence to the 2015 DGA. However, $47 \%$ of participants either met the 2018 PAG (MVPA $\geq 150$ minutes/week) or had higher adherence to the 2015 DGA. The characteristics of participants excluded from the analysis are presented in Supplementary Table 1. Adherence to the 2018 PAG was associated with higher adherence to the 2015 DGA (DGAl-2015 $\geq$ median; odds ratio $0.61,95 \% \mathrm{Cl}, 0.51-0.72 ; \mathrm{p}<.001$ ). For the cross-sectional analysis, $66 \%$ of participants provided 7 days of valid accelerometry data ( $23 \%$ [ 6 days of valid accelerometry data], $8 \%$ [ 5 days of valid accelerometry data], and $3 \%$ [ 4 days of valid accelerometry data]. Similarly, $66 \%, 22 \%, 8 \%$, and $2 \%$ of participants provided $7,6,5$, and 4 days of valid accelerometry data, respectively, at baseline for the longitudinal analysis.

Table 1. Sample Characteristics. 


\begin{tabular}{|c|c|c|}
\hline & Men $(n=1,086)$ & Women $(n=1,293)$ \\
\hline Age (years) & $47 \pm 8$ & $47 \pm 9$ \\
\hline Body mass index $\left(\mathrm{kg} / \mathrm{m}^{2}\right)$ & $28.9 \pm 4.7$ & $26.8 \pm 6.0$ \\
\hline Waist circumference (inches) & $40.2 \pm 4.8$ & $36.1 \pm 5.9$ \\
\hline $\mathrm{SBP}(\mathrm{mm} \mathrm{Hg})$ & $121 \pm 13$ & $113 \pm 14$ \\
\hline $\mathrm{DBP}(\mathrm{mm} \mathrm{Hg})$ & $78 \pm 8$ & $72 \pm 9$ \\
\hline Antihypertensive medication, n (\%) & $222(20.5)$ & $173(13.4)$ \\
\hline Hypertension, n (\%) & $303(28.0)$ & $222(17.2)$ \\
\hline Fasting blood glucose (mg/dL) & $100.3 \pm 19.7$ & $92.4 \pm 13.7$ \\
\hline Diabetes, n (\%) & $63(5.8)$ & $43(3.3)$ \\
\hline Total cholesterol (mg/dL) & $187 \pm 35$ & $187 \pm 35$ \\
\hline Triglycerides (mg/dL) & $129 \pm 83$ & $95 \pm 59$ \\
\hline HDL-C (mg/dL) & $51 \pm 14$ & $67 \pm 18$ \\
\hline LDL-C (mg/dL) & $110 \pm 30$ & $101 \pm 30$ \\
\hline Lipid-lowering medication, n (\%) & $247(22.7)$ & $130(10.1)$ \\
\hline Smoking, n (\%) & $99(9.1)$ & $102(7.9)$ \\
\hline Total caloric intake (kcal/day) & $2,106 \pm 660$ & $1,913 \pm 594$ \\
\hline DGAI-2015 (0-100) & $58.0 \pm 10.7$ & $65.3 \pm 9.7$ \\
\hline Accelerometer wear time (minutes/day) & $934.0 \pm 102.7$ & $911.8 \pm 88.4$ \\
\hline Sedentary time (minutes /day) & $657.8 \pm 70.1$ & $668.9 \pm 67.1$ \\
\hline Light intensity PA (minutes /day) & $129.7 \pm 49.6$ & $123.2 \pm 42.2$ \\
\hline MVPA (minutes /day) & $29.9 \pm 21.5$ & $25.3 \pm 19.9$ \\
\hline Total PA (minutes /day) & $159.6 \pm 59.9$ & $148.5 \pm 51.0$ \\
\hline MVPA $\geq 150$ minutes/week, n (\%) & $641(59.0)$ & $613(47.4)$ \\
\hline MetS, n (\%) & $361(33.2)$ & $223(17.3)$ \\
\hline
\end{tabular}

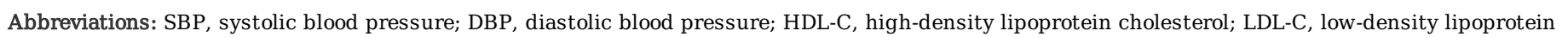

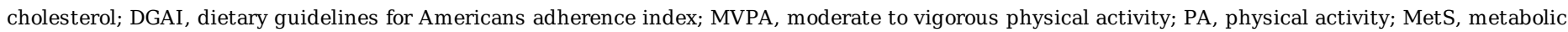
syndrome

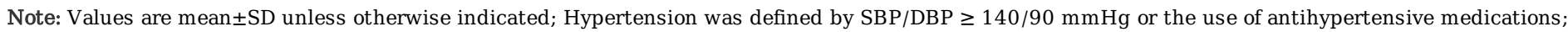
Diabetes was defined as a fasting blood glucose $\geq 126 \mathrm{mg} / \mathrm{dL}$ or use of insulin or oral hypoglycemic agents .

\section{Cross-sectional associations of adherence to the 2018 PAG and 2015 DGA with the prevalence of MetS}

The individual and conjoint cross-sectional associations of adherence to the 2018 PAG and 2015 DGA with the prevalence of MetS are shown in Tables 2 and Fig. 1 (Additional file 1), respectively. The prevalence of MetS was $24.6 \%(n=584 / 2,379)$. After adjusting for covariates, participants who met the 2018 PAG had lower odds of MetS, compared to those who did not. Likewise, participants with higher adherence to the DGA (DGAI-2015 $\geq$ median) had lower odds of MetS, compared to those with poor adherence (DGAI-2015 < median). Results were similar in analyses examining the MVPA and DGAI-2015 as continuous variables (Table 2). Every 10 minutes per day increase in MVPA or 10-point increase in DGAI-2015 was associated with $18 \%$ or $19 \%$ lower odds of prevalent MetS, respectively (Table 2 ).

Table 2. Cross-sectional associations of adherence to the 2018 PAG and 2015 DGA with the presence of MetS. 


\begin{tabular}{|c|c|c|c|c|}
\hline 2018 PAG Adherence & \#MetS/\#total & OR & $95 \% \mathrm{CI}$ & $\mathrm{P}$ value \\
\hline MVPA <150 min/week & $358 / 1,125$ & Referent & & \\
\hline MVPA $\geq 150$ minutes/week & $226 / 1,254$ & 0.49 & $0.40-0.60$ & $<.001$ \\
\hline MVPA (per 10 minutes increment) & $584 / 2,379$ & 0.82 & $0.76-0.88$ & $<.001$ \\
\hline 2015 DGA Adherence & \#MetS/\#total & OR & $95 \% \mathrm{CI}$ & $\mathrm{P}$ value \\
\hline DGAI_2015<median & $342 / 1186$ & Referent & -- & \\
\hline DGAI_2015 <median & $242 / 1193$ & 0.67 & $0.54-0.83$ & $<.001$ \\
\hline DGAI-2015 (per 10 point increment) & $584 / 2,379$ & 0.81 & $0.74-0.89$ & $<.001$ \\
\hline Conjoint association & \# MetS/\#Total & OR & $95 \% \mathrm{CI}$ & $P$ value \\
\hline MVPA $<150 \mathrm{~min} /$ week \& DGAI-2015 <median & $210 / 600$ & Referent & & \\
\hline MVPA $\geq 150 \mathrm{~min} /$ week \& DGAI-2015 <median & $132 / 586$ & 0.57 & $0.43-0.75$ & $<.001$ \\
\hline MVPA $<150 \mathrm{~min} /$ week $\&$ DGAI-2015 $\geq$ median & $148 / 525$ & 0.80 & $0.60-1.06$ & .12 \\
\hline MVPA $\geq 150 \mathrm{~min} /$ week $\&$ DGAI-2015 $\geq$ median & $94 / 668$ & 0.35 & $0.26-0.47$ & $<.001$ \\
\hline
\end{tabular}

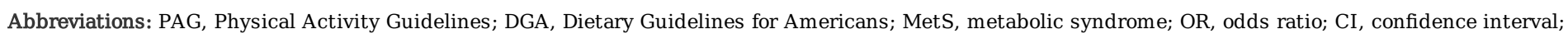
MVPA, moderate to vigorous physical activity; DGAI, dietary guidelines for Americans adherence index.

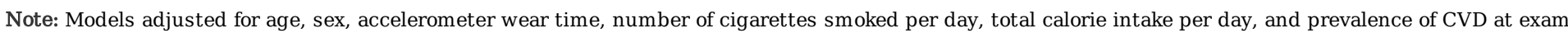
2; Accelerometer wear time was excluded in the model evaluating the association between adherence to the 2015 DGA and presence of MetS; Family

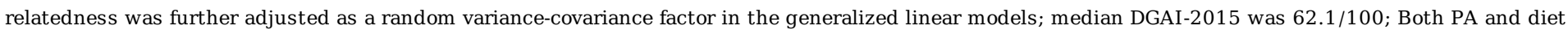
quality were measured at the second examination cycle (2008-2011); Bold P-values indicate statistical significance.

We observed a significant multiplicative interaction between MVPA and DGAI-2015 on the association of each with odds of MetS $\left(P_{\text {interaction }}=0.02\right)$. In the conjoint analysis, we observed that participants who met the 2018 PAG (MVPA $\geq 150$ minutes/week) had lower odds of MetS with higher adherence to the 2015 DGA. Participants who met the 2018 PAG and had higher adherence to the 2015 DGA were less likely to have MetS compared to the referent group (i.e., participants who did not meet the 2018 PAG and had poor adherence to the 2015 DGA; Table 2 and Fig. 1 [Additional file 1]).

\section{Longitudinal associations of adherence to the 2018 PAG and 2015 DGA with the incidence of MetS}

In our sample of participants who were free of MetS at baseline (Sample 2, $n=1,562), 287(18.4 \%)$ individuals developed new-onset MetS over an average follow-up of 8 years. After adjusting for covariates, adherence to the 2018 PAG was associated with a lower risk of developing MetS. Similarly, higher adherence to the 2015 DGA (DGAl-2015 $\geq$ median) was associated with a lower risk of MetS compared to those with poor adherence to the 2015 DGA (DGAl-2015 < median). Additionally, every 10 minutes per day increase in MVPA and each 10-point increase in DGAI-2015 were associated with $8 \%$ and $13 \%$ lower risk of developing MetS, respectively (Table 3).

Table 3. Longitudinal associations of adherence to the 2018 PAG and 2015 DGA with the incidence of MetS. 


\begin{tabular}{lcccc}
\hline 2018 PAG Adherence & \# Events/\# At risk & HR & $95 \%$ CI & P value \\
\hline MVPA $<150$ min/week & $141 / 648$ & \multicolumn{2}{c}{ Referent } \\
MVPA $\geq 150$ min/week & $146 / 914$ & 0.66 & $0.50-0.88$ & .004 \\
\hline MVPA (per 10 minutes increment/day) & $287 / 1,562$ & 0.92 & $0.86-0.99$ & .03 \\
\hline 2015 DGA Adherence & \# Events/\#At risk & HR & $95 \%$ CI & P value \\
\hline DGAI-2015 <median & $174 / 781$ & Referent & .008 \\
DGAI-2015 $\geq$ median & $113 / 781$ & 0.68 & $0.51-0.90$ & .03 \\
\hline DGAI-2015 (per 10 point increment) & $287 / 1,562$ & 0.87 & $0.76-0.99$ \\
\hline Conjoint association & \#Events/\#At risk & HR & $95 \%$ CI & P value \\
\hline MVPA $<150$ min/week \& DGAI-2015 <medain & $86 / 339$ & Referent & & .10 \\
MVPA $\geq 150$ min/week \& DGAI-2015 <median & $88 / 442$ & 0.74 & $0.51-1.06$ \\
MVPA $<150$ min/week \& DGAI-2015 $\geq$ median & $55 / 309$ & 0.78 & $0.52-1.17$ & .22 \\
MVPA $\geq 150$ min/week \& DGAI-2015 $\geq$ median & $58 / 472$ & 0.48 & $0.32-0.70$ & $<.001$ \\
\hline
\end{tabular}

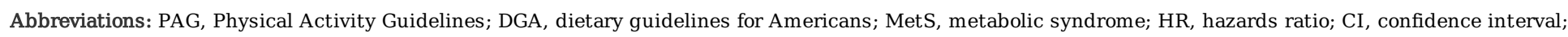
MVPA, moderate to vigorous physical activity; DGAI, diet guidelines for Americans adherence index;

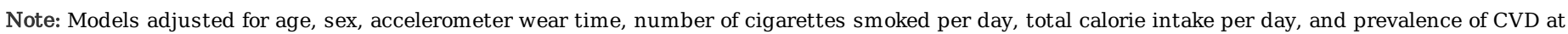

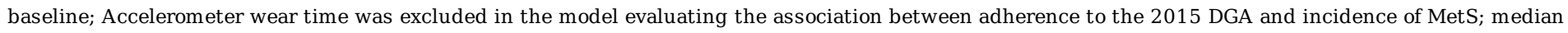

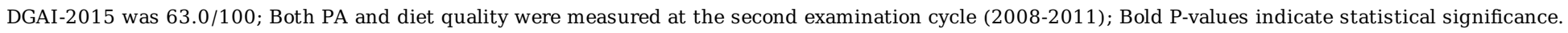

We also observed a significant multiplicative interaction between MVPA and DGAI-2015 on the association of each with the risk of MetS $\left(P_{\text {interaction }}=0.03\right)$. In our longitudinal analysis, participants who met the 2018 PAG and had higher adherence to the 2015 DGA had a lower risk of MetS when compared to the referent group (MVPA $<150$ minutes/week and DGAI-2015 < median; Table 3 and Fig. 1 [Additional file 1]).

\section{Sensitivity Analysis}

In cross-sectional analysis, participants with either second or third tertile of DGAl-2015 had lower odds of MetS, after adjusting for covariates (Supplementary Table 2). Additionally, among participants who met the 2018 PAG (MVPA $\geq 150$ minutes/week), we observed a dose-response relation, with progressively lower odds of MetS as adherence to the 2015 DGA increased (across tertiles of the DGAI-2015). Participants who met the 2018 PAG and had optimal adherence to the 2015 DGA (third tertile of DGAI2015) were $68 \%$ less likely to have MetS compared to participants who did not meet the 2018 PAG and had poor adherence to the 2015 DGA (first tertile of DGAl-2015; Supplementary Table 3 and Supplementary Fig. 1). However, we did not observe an association between either second or third tertiles of DGAl-2015 and risk of MetS compared to the first tertile of the DGAl-2015 prospectively (Supplementary Table 4). Only participants who met the 2018 PAG and had optimal adherence to the 2015 DGA (third tertile of DGAI-2015) had lower risk of Mets when compared to the referent group (MVPA $<150$ minutes/week and first tertile of DGAl-2015; Supplementary Table 5 and Supplementary Fig. 2).

\section{Discussion}

Principal findings 
The primary finding of our investigation is that adherence to both PA and dietary guidelines in middleadulthood is inversely associated with odds of MetS cross-sectionally, and with a lower risk of developing MetS later in life prospectively. Particularly, we observed a dose-response association, in which there were lower odds or risk of MetS for those who met both the 2018 PAG and demonstrated higher ( $\geq$ median) or optimal ( $\geq$ third tertile) adherence to the 2015 DGA, which is suggestive of a potential synergistic effect of PA and diet on cardiometabolic health. Overall, our findings underscore the importance of maintaining both a regular physical activity schedule and following a healthy diet in mid-adulthood in order to lower risk of developing cardiometabolic disease in later life.

\section{Comparison with the literature}

Consistent with the current investigation, numerous cross-sectional studies have reported an inverse association between objectively-assessed MVPA and odds of MetS [8, 9, 21, 22]. Additionally, several longitudinal studies have observed an inverse association between adherence to the 2008 PAG, using self-reported MVPA, and incident MetS across different demographic groups, showing approximately 20$60 \%$ lower risk of MetS [23-25]. Furthermore, a number of studies have reported inverse associations between healthy dietary patterns and MetS. This includes studies evaluating both cross-sectional and longitudinal relations of dietary patterns such as the 2005 DGAI [10, 11], Dietary Approaches to Stop Hypertension (DASH) score [26], Mediterranean Diet Score (MDS) [27-28], and the Alternative Healthy Eating Index (AHEI) [29] with MetS. In particular, adherence to the 2005 DGA was associated with a lower odds of MetS in the Framingham Offspring Cohort, where an interaction in the relation of the 2005 DGAI with MetS by age was observed, with larger effect sizes reported in adults younger than 55 years [10]. The effect size they observed in adults younger than 55 years is consistent with ours (OR:0.57, 95\% Cl:0.360.92 [highest 2005 DGAl quintile vs. lowest DGAl quintile] in the previous report vs. OR: $0.67,95 \% \mathrm{Cl}: 0.54-$ 0.83 [DGAl-2015 $\geq$ median vs. DGAI-2015 < median] or OR:0.58, 95\% Cl: 0.45-0.75 [highest DGAl-2015 tertile vs. lowest DGAI-2015 tertile] in the current investigation). The present investigation adds to the existing literature by using objectively measured MVPA and the most recent guidelines for both PA and diet. These data strengthen current evidence that adherence to both guidelines in mid-adulthood may confer the lowest risk of MetS, which often precedes overt cardiovascular disease.

Evidence is limited with regards to the conjoint association of adherence to both the PAG and DGA with MetS. Prior clinical intervention studies have highlighted the adoption of both a physically active lifestyle and well-balanced diet to improve cardiometabolic health [30]. In particular, a 1-year intervention study among men with MetS demonstrated a strong reduction in the prevalence of MetS in the combined intervention group (67.4\%) compared to a single PA (23.5\%) or dietary intervention (35.3\%) [31].

In accordance with the present investigation, prior cross-sectional studies in Asian populations have reported that the combination of a sedentary lifestyle with poor diet quality is associated with higher odds of MetS [32,33]. Differences between our investigation and these previous reports may be due to the use of self-reported PA data, which could lead to overestimation of PA. Similar to the present investigation, other investigators have reported that non-adherence to the 2008 PAG (MVPA $<150$ minutes/week) and an unhealthy diet (Healthy Eating Index-2005 < 60th percentile) was associated with a 
more than doubling of the odds of MetS compared to adherence to the 2008 PAG (MVPA $\geq 150$ minutes/week) and a healthy diet (Healthy Eating Index $\geq 60$ th percentile) [34]. However, a direct comparison between this study and ours is challenging due to differences in dietary assessment methods (24-hour recall vs. 150-item FFQ) and the type of accelerometer (uniaxial vs. omnidirectional).

There is a lack of evidence regarding the conjoint association of PA and diet quality with the incidence of MetS in a large prospective cohort study. Our results indicate that adherence to both PA and dietary guidelines in middle-adulthood may have synergistic effects on lowering the risk of cardiometabolic disease later in life. Further, we observed that $28 \%$ of participants adhered to both the 2018 PAG (MVPA $\geq 150$ minutes/week) and 2015 DGA (DGAl-2015 $\geq$ median), and 47\% adhered to one of the respective guidelines. Only $19 \%$ of participants adhere to both guidelines, and $67 \%$ adhered to one of the respective guidelines when 2015 DGA was categorized into three groups based on the tertile of DGAI-2015. These data suggest that adherence to both 2018 PAG and 2015 DGA may be a good approach to improve cardiometabolic health for a majority of middle-aged adults.

Previous studies have suggested improved endothelial function, insulin resistance, inflammatory profile, and adiposity measures as plausible underlying biological mechanisms mediating associations of PA and diet quality with MetS $[35,36]$. However, mechanistic links concerning the conjoint effects of PA and diet quality on MetS are not fully understood. Several intervention studies have demonstrated improved lipid profiles and glycemic control with reductions in blood pressure, body weight, fat mass, visceral fat, and mid-thigh muscle fat content in participants undergoing combined healthy diet and PA interventions compared to a single PA or dietary intervention [37-38]. Moreover, from a behavioral perspective, a metaanalysis conducted by Colcombe et al reported that higher PA is associated with improved cognitive function, which leads to better self-regulatory skills and robust goal-oriented behavior [39]. Thus, higher PA may indirectly promote healthier eating habits. Indeed, adherence to the 2018 PAG was associated with higher odds of adherence to the 2015 DGA (DGAl-2015 $\geq$ median) in our investigation. Further studies are warranted to explore the underlying biological or behavioral mechanisms that may explain the conjoint associations of PA and diet quality with occurrence of the MetS.

There are several strengths of our investigation. The FHS Third Generation cohort is a deeply phenotyped large community-based sample of middle-aged adults, which may reduce selection bias with respect to comorbidities. Furthermore, the comprehensive and detailed assessment of CVD risk factors minimizes residual confounding. We measured PA objectively using an accelerometer to reduce measurement bias. The use of the latest dietary guidelines adherence index (DGAl-2015) as a more comprehensive measure of dietary quality is another strength of our investigation. This approach penalized excessive consumption of energy-dense foods, limiting the possibility that their overconsumption could lead to higher scoring [40]. Lastly, we used a cross-sectional and prospective cohort study design rendering our findings more robust. However, there are limitations that should be recognized. The DGAI-2015 was derived using data from the Harvard semi-quantitative 150-item FFQ, which is a self-administered questionnaire. Therefore, non-differential misclassification may affect our results. Additionally, in the present investigation, we observed significant multiplicative interactions only when MVPA and DGAI-2015 
were modeled as continuous variables. However, given that the purpose of the present investigation is to examine the conjoint associations of adherence to the 2018 PAG and the 2015 DGA with MetS and to provide more specific behavioral goals, we evaluated the conjoint associations using categorical variables. All participants in the current investigation were white individuals of European ancestry, limiting the generalizability of our findings to other racial groups. Further studies with a multi-ethnic sample are needed to assess for effect modification by race/ethnicity.

\section{Conclusions}

In the present investigation, conjoint adherence to the 2018 PAG and 2015 DGA was associated with the lowest odds of prevalent MetS and lowest risk of developing MetS later in life. These findings emphasize the importance of maintaining adequate PA and consuming a healthy diet in midlife on cardiometabolic health later in life.

\section{Abbreviations}

MetS, metabolic syndrome; CVD, cardiovascular disease; PAG, physical activity guidelines; MVPA, moderate to vigorous physical activity; DGA, dietary guidelines; DGAl, dietary guidelines for Americans adherence index; FHS, Framingham Heart Study; FFQ, food frequency questionnaire; HDL-C, high-density lipoprotein cholesterol

\section{Declarations}

\section{Acknowledgments}

We acknowledge the dedication of the FHS study participants without whom this research would not be possible.

\section{Funding}

The Framingham Heart Study (FHS) acknowledges the support of contracts NO1-HC-25195, R01HL131029, HHSN268201500001I, 75N92019D00031 and T32 grant 5T32HL125232 from the National Heart, Lung and Blood Institute, and grants 15GPSGC24800006 from the American Heart Association. The accelerometry study was supported by R01AG047645. Dr. Vasan is supported in part by the Evans Medical Foundation and the Jay and Louis Coffman Endowment from the Department of Medicine, Boston University School of Medicine.

\section{Availability of data and materials}

Study data and materials available from the corresponding author on request.

Author Contribution: J.L., M.E.W., and R.S.V conceived the idea for the present investigation. J.L., M.T.B., and M.E.W. drafted the manuscript. J.L., M.E.W., G.T.S., P.T.J., and V.X. contributed to the analysis of data. 
N.S., G.T.S., P.T.J., R.S.V., and V.X. provided critical revision of the manuscript. V.X. are the guarantors of this work and, as such, had full access to all the data in the study and take responsibility for the integrity of the data and the accuracy of the data analysis.

\section{Competing interests}

The authors declare that they have no competing interests.

\section{Consent for publication}

Not applicable

Ethics approval and consent to participate: Ethics approval was obtained from the Institutional Review Board at the Boston University Medical center and all participants provided informed consent.

\section{Author details}

${ }^{1}$ Section of Preventive Medicine and Epidemiology, Boston University School of Medicine, Boston, MA.

${ }^{2}$ Department of Health Sciences, Sargent College of Health and Rehabilitation Sciences, Boston University, Boston, MA. ${ }^{3}$ Department of Medicine, Boston University School of Medicine, Boston, MA. ${ }^{4}$ Section of Endocrinology, Diabetes, Nutrition, and Weight Management, Boston University School of Medicine, Boston MA. ${ }^{5}$ Framingham Heart Study, Framingham, MA. ${ }^{6}$ Nutritional Epidemiology Program, Jean Mayer US Department of Agriculture, Human Nutrition Research Center on Aging at Tufts University, Boston, MA. ${ }^{7}$ Department of Epidemiology, Boston University School of Public Health, Boston, MA.

${ }^{8}$ Section of Cardiovascular Medicine, Department of Medicine, Boston University School of Medicine, Boston, MA. ${ }^{9}$ Center for Computing and Data Sciences, Boston University, Boston, MA. ${ }^{10}$ Department of Biostatistics, Boston University School of Public Health, Boston, MA.

\section{References}

1. Zimmet PZ, Magliano DJ, Herman WH, Shaw JE. Diabetes: a 21st century challenge. The lancet Diabetes endocrinology. 2014;2:56-64.

2. Ford ES, Mokdad AH, Giles WH, Brown DW. The metabolic syndrome and antioxidant concentrations: findings from the Third National Health and Nutrition Examination Survey. Diabetes. 2003;52:234652.

3. Alberti K, Eckel RH, Grundy SM, et al. Harmonizing the metabolic syndrome: a joint interim statement of the international diabetes federation task force on epidemiology and prevention; national heart, lung, and blood institute; American heart association; world heart federation; international atherosclerosis society; and international association for the study of obesity. Circulation. 2009;120:1640-5. 
4. Grundy SM, Cleeman JI, Daniels SR, et al. Diagnosis and management of the metabolic syndrome: an American Heart Association/National Heart, Lung, and Blood Institute scientific statement. Circulation. 2005;112:2735-52.

5. Lloyd-Jones DM, Leip EP, Larson MG, et al. Prediction of lifetime risk for cardiovascular disease by risk factor burden at 50 years of age. Circulation. 2006;113:791-8.

6. Physical Activity Guidelines Advisory Committee. 2018 Physical Activity Guidelines Advisory Committee Scientific Report. Washington, DC: U.S. Department of Health and Human Services; 2018. p. 2018.

7. US Department of Agriculture and US Department of Health and Human Services. Dietary Guidelines for Americans, 2015-2020. Washington, DC: US Government Printing Office; 2015.

8. Amirfaiz S, Shahril MR. Objectively measured physical activity, sedentary behavior, and metabolic syndrome in adults: systematic review of observational evidence. Metabolic syndrome related disorders. 2019;17:1-21.

9. Loprinzi PD, Cardinal BJ. Association between biologic outcomes and objectively measured physical activity accumulated in $\geq 10$-minute bouts and $<10$-minute bouts. American Journal of Health Promotion. 2013;27:143-51.

10. Fogli-Cawley JJ, Dwyer JT, Saltzman E, et al. The 2005 Dietary Guidelines for Americans and risk of the metabolic syndrome. The American journal of clinical nutrition. 2007;86:1193-201.

11. Hosseini-Esfahani F, Jessri M, Mirmiran P, Bastan S, Azizi F. Adherence to dietary recommendations and risk of metabolic syndrome: Tehran Lipid and Glucose Study. Metabolism. 2010;59:1833-42.

12. Splansky GL, Corey D, Yang Q, et al. The third generation cohort of the National Heart, Lung, and Blood Institute's Framingham Heart Study: design, recruitment, and initial examination. American journal of epidemiology. 2007;165:1328-35.

13. Crouter SE, Bassett DR. A new 2-regression model for the Actical accelerometer. British journal of sports medicine. 2008;42:217-24.

14. Troiano RP, Berrigan D, Dodd KW, Masse LC, Tilert T, McDowell M. Physical activity in the United States measured by accelerometer. Medicine Science in Sports Exercise. 2008;40:181-8.

15. Heil DP. Predicting activity energy expenditure using the Actical ${ }^{\circledR}$ activity monitor. Research quarterly for exercise sport. 2006;77:64-80.

16. Rimm EB, Giovannucci EL, Stampfer MJ, Colditz GA, Litin LB, Willett WC. Reproducibility and validity of an expanded self-administered semiquantitative food frequency questionnaire among male health professionals. American journal of epidemiology. 1992;135:1114-26.

17. Feskanich D, Rimm EB, Giovannucci EL, et al. Reproducibility and validity of food intake measurements from a semiquantitative food frequency questionnaire. Journal of the American Dietetic Association. 1993;93:790-6.

18. Willett W. Nutritional epidemiology. Vol 40: Oxford university press; 2012. 
19. Lin $\mathrm{H}$, Rogers $\mathrm{GT}$, Lunetta $\mathrm{KL}$, et al. Healthy diet is associated with gene expression in blood: the Framingham Heart Study. The American journal of clinical nutrition. 2019;110:742-9.

20. Kannel W, Wolf P, Garrison R. Section 34: Some Risk Factors Related to the Annual Incidence of Cardiovascular Disease and Death in Pooled Repeated Biennial Measurements: Framingham Heart Study, 30 Year Follow-Up. Framingham Heart Study. 1987;30.

21. Clarke J, Janssen I. Sporadic and bouted physical activity and the metabolic syndrome in adults. Medicine Science in Sports Exercise. 2014;46:76-83.

22. Dankel SJ, Loenneke JP, Loprinzi PD. The individual, joint, and additive interaction associations of aerobic-based physical activity and muscle strengthening activities on metabolic syndrome. International journal of behavioral medicine. 2016;23:707-13.

23. Carnethon MR, Loria CM, Hill JO, Sidney S, Savage PJ, Liu K. Risk factors for the metabolic syndrome: the Coronary Artery Risk Development in Young Adults (CARDIA) study, 1985-2001. Diabetes care. 2004;27:2707-15.

24. Cheriyath P, Duan Y, Qian Z, Nambiar L, Liao D. Obesity, physical activity and the development of metabolic syndrome: the Atherosclerosis Risk in Communities study. Eur J Cardiovasc Prev Rehabil. 2010;17:309-13.

25. He D, Xi B, Xue J, Huai P, Zhang M, Li J. Association between leisure time physical activity and metabolic syndrome: a meta-analysis of prospective cohort studies. In: Springer; 2014.

26. Pimenta AM, Toledo E, Rodriguez-Diez MC, et al. Dietary indexes, food patterns and incidence of metabolic syndrome in a Mediterranean cohort: The SUN project. Clinical nutrition. 2015;34:508-14.

27. Kesse-Guyot E, Ahluwalia N, Lassale C, Hercberg S, Fezeu L, Lairon D. Adherence to Mediterranean diet reduces the risk of metabolic syndrome: a 6-year prospective study. Nutrition Metabolism Cardiovascular Diseases. 2013;23:677-83.

28. Rumawas ME, Meigs JB, Dwyer JT, McKeown NM, Jacques PF. Mediterranean-style dietary pattern, reduced risk of metabolic syndrome traits, and incidence in the Framingham Offspring Cohort. The American journal of clinical nutrition. 2009;90:1608-14.

29. Akbaraly TN, Singh-Manoux A, Tabak AG, et al. Overall diet history and reversibility of the metabolic syndrome over 5 years: the Whitehall II prospective cohort study. Diabetes care. 2010;33:2339-41.

30. Magkos F, Yannakoulia M, Chan JL, et al. Management of the metabolic syndrome and type 2 diabetes through lifestyle modification. Ann Rev Nutr. 2009;29:223-56.

31. Anderssen S, Carroll S, Urdal P, Holme I. Combined diet and exercise intervention reverses the metabolic syndrome in middle-aged males: results from the Oslo Diet and Exercise Study. Scandinavian journal of medicine science in sports. 2007;17:687-95.

32. Kim YJ, Hwang JY, Kim H, Park S, Kwon O. Diet quality, physical activity, and their association with metabolic syndrome in Korean adults. Nutrition. 2019;59:138-44.

33. He Y, Li Y, Lai J, et al. Dietary patterns as compared with physical activity in relation to metabolic syndrome among Chinese adults. Nutrition Metabolism Cardiovascular Diseases. 2013;23:920-8. 
34. Loprinzi PD, Smit E, Mahoney S. Physical activity and dietary behavior in US adults and their combined influence on health. Mayo Clinic Proceedings. 2014;89:190-198.

35. Esposito K, Marfella R, Ciotola M, et al. Effect of a Mediterranean-style diet on endothelial dysfunction and markers of vascular inflammation in the metabolic syndrome: a randomized trial. Jama. 2004;292:1440-6.

36. Dumortier M, Brandou F, Perez-Martin A, Fedou C, Mercier J, Brun J. Low intensity endurance exercise targeted for lipid oxidation improves body composition and insulin sensitivity in patients with the metabolic syndrome. Diabetes metabolism. 2003;29:509-18.

37. Christ M, lannello $C$, lannello PG, Grimm W. Effects of a weight reduction program with and without aerobic exercise in the metabolic syndrome. International journal of cardiology. 2004;97:115-22.

38. Watkins LL, Sherwood A, Feinglos M, et al. Effects of exercise and weight loss on cardiac risk factors associated with syndrome X. Archives of internal medicine. 2003;163:1889-95.

39. Colcombe S, Kramer AF. Fitness effects on the cognitive function of older adults: a meta-analytic study. Psychological science. 2003;14:125-30.

40. Jessri M, Lou WY, L'Abbé MR. The 2015 Dietary Guidelines for Americans is associated with a more nutrient-dense diet and a lower risk of obesity. The American journal of clinical nutrition. 2016;104:1378-92.

\section{Supplementary Files}

This is a list of supplementary files associated with this preprint. Click to download.

- AdditionalFile.pdf

- STROBEchecklistv4cohort.pdf 